\title{
Propagation and Stability of Wavelike Solutions of Finite Difference Equations with Variable Coefficients
}

\author{
M. B. Giles* and W. T. Thompkins, $\mathrm{JR}^{\dagger}{ }^{\dagger}$ \\ Massachusetts Institute of Technology, \\ Cambridge, Massachusetts 02139
}

Received May 25, 1983; revised December 20, 1983

\begin{abstract}
An asymptotic approach is used to analyze the propagation and dissipation of wavelike solutions to finite difference equations. It is shown that to first order the amplitude of a wave is convected at the local group velocity and varies in magnitude if the coefficients of the finite difference equation vary. Asymptotic boundary conditions coupling the amplitudes of different wave solutions are also derived. Equations are derived for the motion of wavepackets and their interaction at boundaries. Comparison with numerical experiments demonstrates the success and limitations of the asymptotic approach. Finally, a global stability analysis is developed. 1985 Academic Press, Inc.
\end{abstract}

$$
\begin{array}{rlrl} 
& \text { Notation } \\
\delta_{x} U_{j+1 / 2} & =U_{j+1}-U_{j} & \mu_{x} U_{j+1 / 2} & =\frac{1}{2}\left(U_{j+1}+U_{j}\right) \\
\Delta_{x} U_{j} & =U_{j+1}-U_{j} & \nabla_{x} U_{j}=U_{j}-U_{j-1} \\
E_{m x} U_{j} & =U_{j+m} & &
\end{array}
$$

When there are several independent variables the subscript on the finite operator denotes the direction of the shift, differencing or averaging. For example,

$$
\begin{aligned}
\text { if } U_{j}^{n} & =u\left(x_{j}, t_{n}\right) \quad \text { then } \delta_{x} U_{j+1 / 2}^{n}=U_{j+1}^{n}-U_{j}^{n} . \\
\bar{A} & =\text { complex conjugate of } A \\
\operatorname{Re}(A) & =\text { Real component of } A \\
\operatorname{Im}(A) & =\operatorname{Imaginary} \text { component of } A
\end{aligned}
$$

* Research Assistant, Dept. of Aeronautics and Astronautics.

${ }^{\dagger}$ Associate Professor, Dept. of Aeronautics and Astronautics. 


\section{INTRODUCTION}

Methods for analyzing dispersive partial differential equations are well established. Using Fourier decomposition and asymptotic evaluation of integrals, or by direct asymptotic expansion $[5,9]$, it can be shown that the encrgy propagates at the local group velocity. Ray theory $[5,9]$ then treats wavepackets, localized wavelike disturbances, as particles and derives simple o.d.e.'s for their motion. This paper applies the techniques to the analysis of numerical wave propagation in finite difference equations. Due to the discretization the numerical waves are always dispersive even if the analytic system being modeled is nondispersive. Until recently the importance of the group velocity in analyzing finite difference solutions does not seem to have been recognized. Kentzer [4] discusses the role of group velocity and shows that in many common schemes the numerical group velocity at high wavenumbers is in the opposite direction to the analytic group velocity. Vichnevetsky and Bowles [8] derive reflection coefficients for the interaction of waves at boundaries, and present several illustrative numerical examples. Trefethen [6] provides a group velocity interpretation of the stability theory of Gustafsson et al. [3], and in a forthcoming paper [7] will derive rigorous conditions for the $P$ stability [10] of two-boundary problems. In the stability analysis in this paper we use $P$-stability, which is concerned with stability in the limit $t \rightarrow \infty$, rather than GKS-stability, which is concerned with stability in the limit $\Delta t \rightarrow 0$. Reference [2] contains further details and numerical examples of the work in this paper. It also includes a more general global stability analysis which allows for variable coefficients in the finite difference equations, and in the case of constant coefficients reduces to the exact stability analysis of Beam et al. [1].

The approach we use is an asymptotic one in which a wave solution is expressed as a product of a complex amplitude and an oscillatory phase function whose frequency and wavenumber may also be complex. The asymptotic assumption, or approximation, is that the length scale for variations in the amplitude and wavenumber is large relative to a mesh cell length. An asymptotic expansion leads to a local dispersion relation relating the wavenumber to the frequency. The firstorder terms produce an equation for the amplitude in which the local group velocity appears as the velocity of convection of the amplitude. Also there is a variation in the magnitude of the amplitude if the coefficients of the finite difference equation vary. All of the wave solutions with a given frequency and different wavenumbers are coupled at the boundaries by asymptotic boundary conditions. If there are only two waves per frequency then this reduces to the amplitude reflection coefficients computed by Vichnevetsky and Bowles [8].

The following section develops a theory for the motion of wavepackets which are wavelike disturbances of finite length and constant frequency. Using the techniques of classical ray theory $[5,9]$, these can be treated as particles and simple o.d.e.'s can be derived to describe their motion and the change in their energy. When they reach the boundary they are reflected into wavepackets of a different wavenumber but the same frequency and the energy of the reflected wavepacket can be 
calculated from boundary reflection coefficients. The last section derives a global stability analysis in which the usual Fourier stability analysis is modified to calculate the effects of nonperiodic boundary conditions and slowly varying coefficients. This analysis is then used to calculate the spectral radius of the backward Euler method.

\section{Asymptotic Amplitude Analysis}

Asymptotic Amplitude Equation

Consider a general linear homogeneous finite difference equation with variable coefficients,

$$
L_{j} U_{j}^{n}=0
$$

where

$$
L_{j} \equiv \sum_{m, p} C_{m p}(x) E_{m x} E_{p l}
$$

If the coefficients $C_{m p}$ are constants then

$$
U_{j}^{n}=\exp [i(j \phi-n \Omega)]
$$

is an exact solution of (1) provided

$$
\sum_{m, p} C_{m p} \exp [i(m \phi-p \Omega)]=0 .
$$

This equation is called the dispersion relation between wavenumber $\phi$ and frequency $\Omega$. If the coefficients are not constant then $U$ can be expressed as

$$
U_{j}^{n}=A(j, n) \operatorname{cxp}\left[i \Psi^{\prime}(j, n)\right]
$$

where $A(j, n)$ is a slowly varying amplitude and $\Psi(j, n)$ is the phase of the wave and is related to the frequency $\Omega$ and wavenumber $\phi$ by

$$
\frac{\partial \Psi}{\partial n}=-\Omega, \quad \frac{\partial \Psi}{\partial j}=\phi
$$

The asymptotic approximation which is made is that the length scale $L_{A}$ and time scale $T_{A}$ for variations in $A$ and the length scale $L_{\phi}$ for variations in $\phi$ are much greater than 1. Substituting (5) into (2) and expanding $A$ and $\Psi$ in Taylor series about a point $(j, n)$ yields 


$$
\begin{aligned}
L_{j} U_{j}^{n}= & \exp [i \Psi] \sum_{m, p} C_{m p}(j) \exp [i(m \phi-p \Omega)] \\
& \times\left(A+m \frac{\partial A}{\partial j}+p \frac{\partial A}{\partial n}+\frac{i m^{2}}{2} A \frac{\partial \phi}{\partial j}\right) \\
& +O\left(A L_{A}^{-2}, A T_{A}^{-2}, A L_{\phi}^{-2}\right) .
\end{aligned}
$$

To satisfy Eq. (1) the amplitude $A(j, n)$ must satisfy

$$
a_{0}(\phi, \Omega, j) A+a_{1}(\phi, \Omega, j) \frac{\partial A}{\partial n}+a_{2}(\phi, \Omega, j) \frac{\partial A}{\partial j}+a_{3}(\phi, \Omega, j) A \frac{\partial \phi}{\partial j}=0
$$

where

$$
\begin{aligned}
& a_{0}(\phi, \Omega, j)=\sum_{m, p} C_{m p}(j) \exp [i(m \phi-p \Omega)] \\
& a_{1}(\phi, \Omega, j)=i\left(\frac{\partial a_{0}}{\partial \Omega}\right)_{\phi, j \text { const }} \\
& a_{2}(\phi, \Omega, j)=-i\left(\frac{\partial a_{0}}{\partial \phi}\right)_{\Omega, j \text { const }} \\
& a_{3}(\phi, \Omega, j)=-\frac{i}{2}\left(\frac{\partial^{2} a_{0}}{\partial \phi^{2}}\right)_{\Omega, j \text { const }}
\end{aligned}
$$

Because of the asymptotic assumptions (8) can only be satisfied if

$$
a_{0}(\phi, \Omega, j)=0+O\left(L_{\phi}^{-1}, L_{A}^{-1}, T_{A}^{-1}\right) .
$$

This is the asymptotic form of the dispersion relation between $\phi$ and $\Omega$ and will usually be satisfied by setting $a_{0}$ identically equal to zero. $\phi$ is now a slowly varying function of $j$ due to the slow variation in the coefficients. Neglecting the second-order terms and dividing by $a_{1}$ gives the asymptotic amplitude equation,

$$
\frac{\partial A}{\partial n}+r_{g} \frac{\partial A}{\partial j}=\varepsilon A
$$

where

$$
r_{g}=a_{2} / a_{1} \quad \text { and } \quad \varepsilon=-\left(a_{3} \frac{\partial \phi}{\partial j}+a_{0}\right) / a_{1} .
$$

Differentiating Eq. (10) with $j$ held constant gives

$$
d a_{0}=\left(\frac{\partial a_{0}}{\partial \Omega}\right)_{\phi, j \text { const }} d \Omega+\left(\frac{\partial a_{0}}{\partial \phi}\right)_{\Omega, j \text { const }} d \phi=0 .
$$


Hence,

$$
\left(\frac{\partial \Omega}{\partial \phi}\right)_{j \mathrm{const}}=a_{2} / a_{1}=r_{\mathrm{g}} .
$$

Thus the amplitude $A$ of the wave is convected at the local group velocity.

\section{Asymptotic Boundary Conditions}

The general solution of Eq. (1) is a sum of waves with different constant frequencies $\Omega$ and slowly varying wavenumber $\phi$ and amplitude $A$.

$$
U_{j}^{n}=\sum_{\Omega} \sum_{m=1}^{M} A_{m}(j, n) \exp \left[i\left(\int_{0}^{j} \phi_{m}(\xi) \delta \xi-n \Omega\right)\right] .
$$

The outer summation is over diffcrent values of $\Omega$, and the inner summation is over the $M$ different values of $\phi$ which satisfy the dispersion relation for each $\Omega$. For each $\Omega, m$ the amplitude $A$ satisfies the asymptotic amplitude equation on the interior of the computational domain independent of all the other waves. All the waves of each frequency are, however, coupled by boundary conditions.

Suppose one of the finite difference boundary conditions at $j=J$ is

$$
B U_{J}^{n} \equiv \sum_{l, p} D_{l p} E_{l x} E_{p t} U_{J}^{n}=0 .
$$

Performing the same expansion as in the derivation of the asymptotic amplitude equation, retaining only the leading terms, and equating the coefficients of $\exp (-i \Omega)$ for each $\Omega$, the boundary condition becomes

$$
\sum_{m=1}^{M} b\left(\Omega, \phi_{m}\right) A_{m}(J) \exp \left[i \int_{0}^{J} \phi_{m} d \xi\right]=0
$$

where

$$
b\left(\Omega, \phi_{m}\right)=\sum_{l, p} D_{l p} \exp \left(i\left(l \phi_{m}-p \Omega\right)\right) .
$$

There are similar boundary conditions at $j=0$.

\section{Ray Theory And Wavepacket Particles}

In addition to the approximations made earlier this section assumes that for all real wavenumbers $\phi$, the frequency $\Omega$ is real for all $j$ and so the group velocity $r_{g}$ is real. 
A Lagrangian-type total time derivative is defined by

$$
\frac{d}{d n} \equiv \frac{\partial}{\partial n}+r_{g} \frac{\partial}{\partial j}
$$

so

$$
\begin{aligned}
& \frac{d j}{d n}=r_{g} \\
& \frac{d A}{d n}=\varepsilon A
\end{aligned}
$$

and

$$
\frac{d \phi}{d n}=r_{g} \frac{\partial \phi}{\partial j}
$$

A general initial value problem for a wave of frequency $\Omega$ and wave-number $\phi(\Omega, j)$ can be solved by integrating these equations from given initial conditions.

A wavepacket is a wave for which the amplitude $A$ is nonzero on only a small part of the domain. The energy is defined to be

$$
E(n)=\int_{x_{0}}^{x_{J}}\left|A\left(x, t_{n}\right)\right|^{2} d x=\int_{0}^{J}|A(j, n)|^{2} \frac{d x}{d j} d j .
$$

Differentiating this definition, and using (22), yields

$$
\frac{d E}{d n} \simeq\left[\varepsilon+\bar{\varepsilon}+\left(\frac{d x}{d j}\right)^{-1} \frac{\partial}{\partial j}\left(r_{g} \frac{d x}{d j}\right)\right] E .
$$

Thus Eqs. (21) and (25) describe the motion of a wavepacket particle in the interior of the computational domain. When the wavepacket reaches a boundary it is reflected as one or more wavepackets with the same frequency but different wavenumber. For the case in which there are just two wavenumbers corresponding to the same frequency the ratio of the reflected energy $E_{2}$ to the incident energy $E_{1}$ is given by

$$
\frac{E_{2}}{E_{1}}=\left|\frac{r_{g}\left(\phi_{2}, J\right)}{r_{g}\left(\phi_{1}, J\right)}\right|\left|R_{J}\right|^{2}
$$

where the amplitude reflection coefficient $R_{J}$ is defined by

$$
R_{J}=\frac{A_{2}(J, n)}{A_{1}(J, n)}
$$

and is determined from the asymptotic boundary condition (18). 
EXAMPLE. The example is the solution of the model convective equation,

$$
\frac{\partial u}{\partial t}+c \frac{\partial u}{\partial x}=0
$$

using a trapezoidal method,

$$
\left(\delta_{t}+\frac{1}{2} r_{j+1 / 2} \mu_{t} \Delta_{x}+\frac{1}{2} r_{j-1 / 2} \mu_{t} \nabla_{x}\right) U_{j}^{n \mid 1 / 2}=0
$$

with

$$
r_{j+1 / 2}=\frac{c \Delta t}{x_{j+1}-x_{j}} .
$$

Figure 1 shows the solution $U(x, t)$ corresponding to a uniform grid $0<j<200$ with $r=1$, and initial conditions corresponding to a wavepacket approximately 20 mesh units wide. Comparison of the heights of the wavecrests a-e at time levels 60 and 120 shows that the phase velocity, the velocity of the wavecrests, is greater than the group velocity, the velocity of the wavepacket.

Figures 2 and 3 show comparisons of the wavepacket theory with numerical experiments. In each case "experimental" values for $X(n)$, the position of the wavepacket, and $E(n)$, its energy, are obtained by solving the finite difference equations and "predicted" values are calculated by solving the wavepacket equations. The initial wavepacket in each case is similar to that in the previous example.

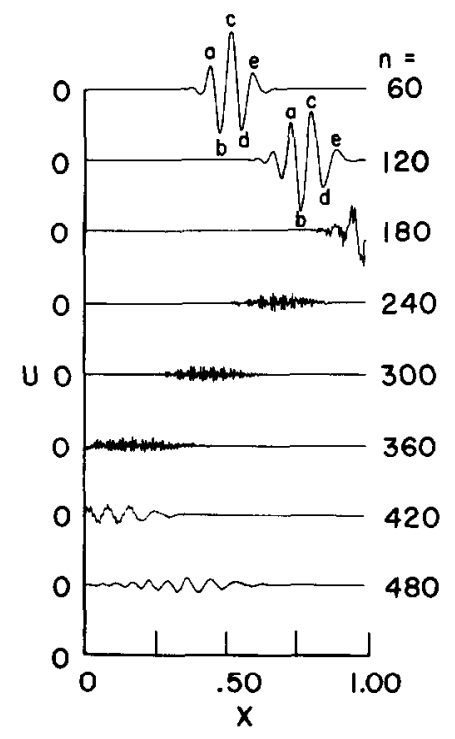

FIG. 1. Numerical solution of convection equation. 


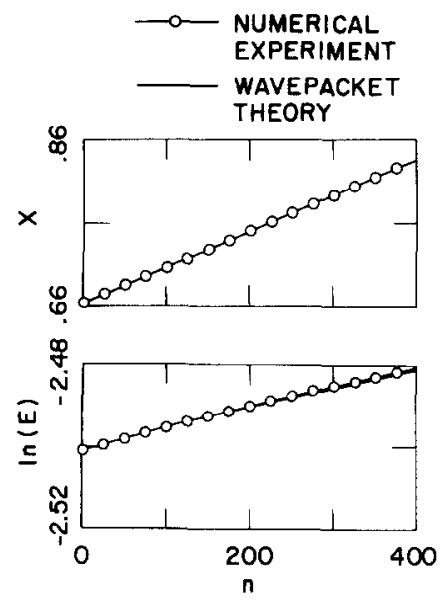

FIG. 2. Position and energy of wavepacket: effect of nonconstant CFL number.

In the first case $r$ varies exponentially from 0.05 at $j=0$ to 0.2 at $j=200$ and $\Omega=0.04$. Figure 2 shows $X(n)$ and $\ln [E(n)]$ both predicted and experimental. This example shows the movement of a wavepacket and the change in its energy due to the variation in $r$. The agreement between the predicted and experimental values is excellent. The energy of the analytic solution is constant so the wavepacket theory has successfully predicted almost all of the change in the numerical cnergy duc to the nonuniform grid.

In the second case $r$ is constant and equal to 1.0 and $\Omega=0.3$. Figure 3 shows $X(n)$ and $\ln [E(n)]$. This example illustrates the effect of the downstream boundary

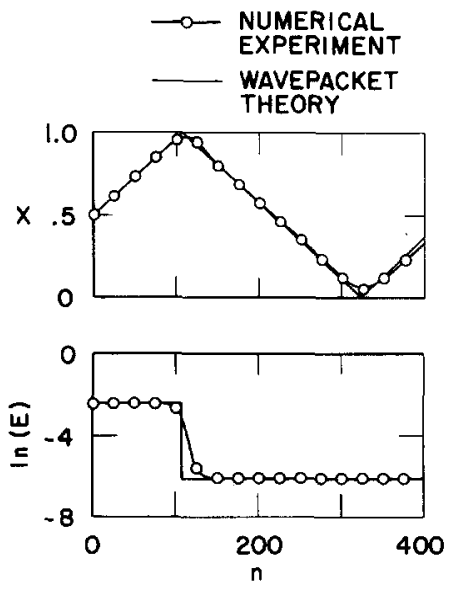

Fig. 3. Position and energy of wavepacket: reflections at boundaries. 
reflecting a wavepacket with reduced energy. Because of the finite length of the wavepacket the drop in energy is smeared and $X(n)$ does not quite reach 1.0. Again the agreement is excellent with the wavepacket theory accurately predicting the energy of the reflected wavepacket.

\section{Asymptotic Stability Analysis}

In this section it is assumed that there are two wavenumbers corresponding to each frequency, and that if one is real then so too is the other. Examples of methods satisfying these conditions are the trapezoidal method applied to the model convective problem with variable CFL number $r$, and the whole class of Beam-Warming schemes applied to the model convective problem with constant CFL number.

The normal Fourier analysis assumes constant coefficients and periodic boundary conditions and derives eigenfrequencies $\Omega(\phi)$ where $\phi$ is a real wavenumber satisfying the periodic boundary conditions and $\Omega(\phi)$ is the corresponding frequency given by the dispersion relation. The common use of Fourier analysis to predict the stability of problems with nonperiodic boundary conditions implicitly assumes that $\Omega(\phi)$ is a close approximation to the true eigenfrequency. This section follows that assumption, calculates a correction $\Omega^{\prime}$ to the Fourier frequency $\Omega(\phi)$ due to the boundary conditions, and then determines the validity of the assumption based on the asymptotic errors.

$$
U_{j}^{n}=A_{1}(j, n) \exp \left(i \int_{0}^{j} \phi_{1} d \xi-i n \Omega\right)+A_{2}(j, n) \exp \left(i \int_{0}^{j} \phi_{2} d \xi-i n \Omega\right)
$$

If the true eigenfrequency is $\Omega+\Omega^{\prime}$ then $A \propto \exp \left(-i n \Omega^{\prime}\right)$ and so

$$
A_{m}(j, n)=\exp \left(-i n \Omega^{\prime}\right) A_{m}(j, 0), \quad m=1,2 .
$$

Substituting these expressions into the asymptotic amplitude equations to evaluate the time derivative and then integrating the resultant o.d.e. gives

$$
A_{m}(J, 0)=A_{m}(0,0) \exp \left(\int_{0}^{J}\left(\frac{\varepsilon+i \Omega^{\prime}}{r_{g}}\right)_{m} d j\right), \quad m=1,2 .
$$

The two asymptotic boundary conditions then become in matrix form

$$
B\left(\begin{array}{l}
A_{1}(0,0) \\
A_{2}(0,0)
\end{array}\right)=0 .
$$

A nontrivial solution exists only if $\operatorname{det} B=0$ and this leads to the following equation for $\Omega^{\prime}$ : 


$$
\begin{aligned}
\exp \left(i \Omega^{\prime} \int_{0}^{J}\left[r_{g}\left(\phi_{1}, j\right)\right]^{-1}-\left[r_{g}\left(\phi_{2}, j\right)\right]^{-1} d j\right) \\
\quad=\frac{b_{2}\left(\Omega, \phi_{2}\right) b_{2}\left(\Omega, \phi_{1}\right)}{b_{2}\left(\Omega, \phi_{1}\right) h_{1}\left(\Omega, \phi_{2}\right)} \exp \left(i \int_{0}^{J} \phi_{2}-\phi_{1} d j\right) \exp \left(\int_{0}^{J}\left(\frac{\varepsilon}{r_{g}}\right)_{2}-\left(\frac{\varepsilon}{r_{g}}\right)_{1} d j\right) .
\end{aligned}
$$

If $\phi_{1}, \phi_{2}$ are chosen so that the r.h.s. is real and positive then

$$
\Omega^{\prime}=-\frac{i}{N}\left(\log \left|\frac{b_{2}\left(\Omega, \phi_{2}\right) b_{1}\left(\Omega, \phi_{1}\right)}{b_{2}\left(\Omega, \phi_{1}\right) b_{1}\left(\Omega, \phi_{2}\right)}\right|+\operatorname{Re} \int_{0}^{J}\left(\frac{\varepsilon}{r_{g}}\right)_{2}-\left(\frac{\varepsilon}{r_{g}}\right)_{1} d j\right)
$$

where

$$
N=\int_{0}^{J}\left[r_{g}\left(\phi_{1}, j\right)\right]^{-1}-\left[r_{g}\left(\phi_{2}, j\right)\right]^{-1} d j .
$$

Thus the frequency $\Omega$ resulting from a normal Fourier analysis is corrected by an amount $\Omega^{\prime}$ due to boundary conditions and variable coefficients. This approach, using $\Omega$ as an initial approximation to the actual eigenfrequency, is valid provided the asymptotic error is small. The asymptotic error is $O\left(L_{A}^{-2}, T_{A}^{-2}\right)=O\left(J^{-2}, \Omega^{\prime 2}\right)$ so provided $r_{g} \ll J, N \gg 1$ and hence $\Omega^{\prime} \ll 1$ except near frequencies for which

$$
\left|\frac{b_{2}\left(\Omega, \phi_{2}\right) b_{1}\left(\Omega, \phi_{1}\right)}{b_{2}\left(\Omega, \phi_{1}\right) b_{1}\left(\Omega, \phi_{2}\right)}\right|
$$

is zero, or infinite, which usually occurs at $\Omega=0$. However, these frequencies are heavily damped by the boundary conditions and so an accurate estimate of their eigenfrequencies is not essential. This method gives accurate asymptotic values near the critical frequencies which are least damped and which therefore determine the overall spectral radius of the scheme.

EXAMPLE. This example is the backward Euler method applied to the model convective problem with constant CFL number $r$ and space extrapolation at the downstream boundary. The finite difference equation is

$$
\left(\nabla_{t}+r \mu_{x} \delta_{x}\right) U_{j}^{n+1}=0
$$

and the dispersion relation is

$$
a_{0}=1-\exp (i \Omega)+i r \sin (\phi)=0 .
$$

After carrying out the calculations the frequency correction $\Omega^{\prime}$ is found to be [2]

$$
\Omega^{\prime}=-\frac{i r \cos (\phi)(1-i r \sin (\phi))}{2 J\left(1+r^{2} \sin ^{2}(\phi)\right)} \log [\cot (\phi / 2)] .
$$


Thus the effect of the boundary conditions is to greatly accelerate convergence at low wavenumbers while having little effect on the higher wavenumbers. The spectral radius $\lambda$ is

$$
\begin{aligned}
\lambda & =\max _{\phi}\left|\exp \left(-i\left(\Omega+\Omega^{\prime}\right)\right)\right| \\
& \simeq 1-\frac{r \log (J)}{4 J} \quad \text { for } J \gg r .
\end{aligned}
$$

\section{Conclusions}

The validity of the asymptotic approach developed in this paper is demonstrated by the numerical results in Section III. The limitations of the wavepacket theory are due to the asymptotic approximations involved in treating the wavepacket as a particle. The stability analysis in Section IV uses fewer approximations and so the errors will be substantially smaller.

The calculation of the asymptotic amplitude equation and boundary conditions for a particular case is no more difficult than a normal Fourier analysis. For applicable cases the wavepacket theory and the stability analysis are straightforward. In more complex cases the main benefit from the theory is the insight given by the asymptotic amplitude equation and boundary conditions. The amplitude equation gives the group velocity and the effect of varying coefficients which is of great interest since in 2-D cascade geometries cell lengths can vary by factors of up to 100 in inviscid calculations and up to 1000 in viscous calculations. The asymptotic boundary conditions give the amplitude reflection coefficients which provide a practical criterion for choosing the best numerical boundary conditions.

\section{ACKNOWLEDGMENTS}

This research was supported by NASA Lewis Research Center under Grant NAG3-9 with Technical Monitor Dr. R. V. Chima, and by a Scholarship for M. Giles from the Kenedy Memorial Trust.

\section{REFERENCES}

1. R. BEAm, R. WARming, AND H. YeE, Stability analysis for numerical boundary conditions and implicit difference approximations of hyperbolic equations, in "Proceedings, NASA Symposium on Numerical Boundary Procedures, 1981," pp. 99-207.

2. M. Giles AND W. T. ThOMPKins, JR., "Asymptotic Analysis of Numerical Wave Propagation in Finite Difference Equations," MIT Gas Turbine and Plasma Dynamics Laboratory Report No. 171, February 1983.

3. B. Gustafsson, H-O. Kriess, and A. Sundstrom, Math. Comp. 26 (1972), 649-686.

4. C. P. Kentzer, "Group Velocity and Propagation of Numerical Errors," AIAA Paper No. 72-153. 
5. J. Lighthill, "Waves in Fluids," pp. 237-260, Cambridge Univ. Press, London/New York, 1978.

6. L. N. Trefethen, J. Comput. Phys. 49 (1983), 199-217.

7. L. N. Trefethen, Stability of finite difference Models Containing Two Boundaries or Interfaces," ICASE Report No. 84-11, NASA CR 172319, March 1984; submitted for publication.

8. R. ViCHNEVETSKY AND J. Bowles, "Fourier Analysis of Numerical Approximations of Hyperbolic Equations," Studies in Applied Mathematics, SIAM, Philadelphia, 1982.

9. G. B. Whitham, "Linear and Nonlinear Waves," Chap. 11, Wiley, New York, 1974.

10. H. YEE, R. BEAM, AND R. WARMING, "Stable Boundary Approximations for a Class of Implicit Schemes for the One-Dimensional Inviscid Equations of Gas Dynamics," AIAA-81-1009-CF, AIAA Computational Fluid Dynamics Conference, Palo Alto, June 22-23, 1981. 